\title{
Impedimetric Biosensor to Enable Fast Evaluation of Gaseous Sterilization Processes ${ }^{+}$
}

\author{
Jan Oberländer 1,2, Julio Arreola 1,2, Christina Hansen ${ }^{1}$, Anton Greeff ${ }^{3}$, Marlena Mayer ${ }^{3}$, \\ Michael Keusgen ${ }^{2}$ and Michael J. Schöning 1,4,* \\ 1 Institute of Nano- and Biotechnologies (INB), FH Aachen University of Applied Sciences, \\ Heinrich-Mußmann-Str. 1, 52428 Jülich, Germany; oberlaender@fh-aachen.de (J.O.); \\ arreola@fh-aachen.de (J.A.); christina.hansen@alumni.fh-aachen.de (C.H.) \\ 2 Institute of Pharmaceutical Chemistry, Philipps-University Marburg, Marbacher Weg 6-10, 35037 Marburg, \\ Germany; keusgen@staff.uni-marburg.de \\ 3 Elopak EQS GmbH, Hanns-Martin-Schleyer-Str. 17, 41199 Mönchengladbach, Germany; \\ anton.greeff@elopak.com (A.G.); marlena.mayer@elopak.com (M.M.) \\ 4 Peter-Grünberg Institute (PGI-8), Forschungszentrum Jülich GmbH, 52428 Jülich, Germany \\ * Correspondence: schoening@fh-aachen.de; Tel.: +49-241-600-953-215 \\ + Presented at the Eurosensors 2017 Conference, Paris, France, 3-6 September 2017.
}

Published: 8 August 2017

\begin{abstract}
Sterilization of packages prior to product filling is a key step in aseptic filling machines. Chemical sterilization is one of the main conventional techniques in many packaging industries. To monitor the effect of sterilization on test microorganisms (Bacillus atrophaeus spores), an impedimetric sensor approach was developed based on a planar interdigitated electrode (IDE) design. In this work, sensor measurements were conducted to prove sensor functionality at different hydrogen peroxide concentrations.
\end{abstract}

Keywords: interdigitated electrodes; gaseous sterilization processes; hydrogen peroxide; microbiological spores; aseptic filling

\section{Introduction}

Aseptic filling of sensitive food products, such as milk or low-acid juices, has become the technology of choice to ensure extended product shelf-life and consumer-safe products. In this process the package material and goods are separately sterilized. Heat-treatment technologies are applied to sterilize the goods, whereas a chemical sterilization is applied for the package material. High-temperature diluted hydrogen peroxide $\left(\mathrm{H}_{2} \mathrm{O}_{2}\right)$ gas is one of the common sterilizing agents in food packaging industries due to its strong oxidizing effects, which possess germicidal and sporicidal properties [1,2]. The efficiency of the sterilization process is evaluated by laborious microbiological challenge tests (count-reduction or end-point-test), which require transferring and processing of samples in a lab. Due to statistical fluctuations of the microbiological methods high numbers of samples are necessary. The microbiological samples are transferred aseptically into nutrition medium, after incubation at predefined temperature; the outgrown colonies can be counted. The efficiency of a sterilization process is defined as ratio between the initial number of organisms and number of surviving organisms after sterilization. The time delay between the sterilization process and lab results lead to possible product damages; thereby, storage of filled products is necessary to prevent product recalls. In order to reduce the laboratory workload a sensor-based method is preferable, to shorten the time delay after the process. In recent works, a sensor consisting of interdigitated electrodes has been developed to electrically characterize the impact of the sterilization process on immobilized, resilient test microorganism, such as spores of 
Bacillus atrophaeus [3,4]. The spore of this microorganism is applied to microbiologically evaluate sterilization processes in industry [5].

\section{Materials and Methods}

\subsection{Sensor Fabrication}

The sensor, presented in Figure 1, is based on a differential set-up of two IDEs. One IDE-sensor structure serves as a reference element, while on the second IDE-sensor structure, microbiological spores of B. atrophaeus are immobilized. The sensor has been designed and fabricated in-house. As sensor-base a glass substrate Borofloat 33 (Schott $\mathrm{GmbH}$ ) has been chosen to reduce parasitic capacitances as known for semiconducting materials combined with insulation layers. To fabricate the interdigitated electrodes conventional photolithography process was applied. The interdigitated electrodes are designed on a glass mask with dimensions of finger width and interspacing of $5 \mu \mathrm{m}$, respectively. The finger length is designed to $3.25 \mathrm{~mm}$; by a number of 614 fingers a sensing area of about $20 \mathrm{~mm}^{2}$ is achieved. As electrode materials either a combination of $10 \mathrm{~nm}$ chromium and $100 \mathrm{~nm}$ gold or $10 \mathrm{~nm}$ titanium and $100 \mathrm{~nm}$ platinum has been used, whereby the $10 \mathrm{~nm}$ layer serves as adhesion promoter between the glass substrate and the respective second metal layer.

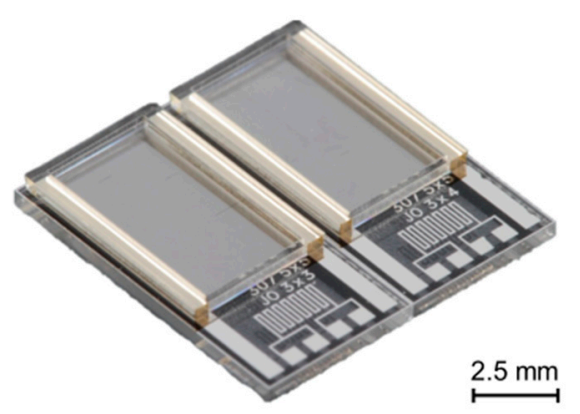

Figure 1. Photograph of the differential sensor set-up of two IDE arrays, consisting of two independent sensing areas of $20 \mathrm{~mm}^{2}$.

\subsection{Impedimetric Characterization}

The electrical characterizations of the IDE arrays are performed with an Agilent E4980A impedance analyzer. A customized measurement set-up has been built up to enable a fast connection of the sensors in vacuo to exclude environmental influences such as humidity variations. An alternating voltage of $20 \mathrm{mV}$, around a $0 \mathrm{~V}$ bias voltage, was applied between the electrode terminals. The resulting current is used to determine the impedance and phase of each sensor. A set of two IDEs is utilized to evaluate the sterilization process. On one of the IDEs microbiological spores of B. atrophaeus are immobilized. The second IDE is left blank to serve as a reference.

\subsection{Sterilization Process}

The sensors are sterilized on a previously designed test-rig, which is a representative of the sterilization module used on industrial aseptic filling machines [6]. The $\mathrm{H}_{2} \mathrm{O}_{2}$ vapor is generated from an aqueous $\mathrm{H}_{2} \mathrm{O}_{2}$ solution $(35 \% w / w)$ and is fed into an air stream as carrier gas. The air- $\mathrm{H}_{2} \mathrm{O}_{2}$ mixture is vaporized at high temperature and is then guided into the sterilization chamber. The sensors are exposed via a pneumatic slide into the vapor stream. In the following experiments the gas temperature was set to $240{ }^{\circ} \mathrm{C}$ and the air-flow was adjusted to $10 \mathrm{~m}^{3} / \mathrm{h}$.

\subsection{Surface Characterisation}

Scanning electron microscopy (SEM) on a Jeol JSM-7800F was applied to study the effect of the sterilization process on the applied test microorganisms. 


\section{Results and Discussion}

\subsection{Impedimetric Results}

In Figure 2, the Bode plot of the impedance characterization of the biosensor chip is shown. The black lines represent the blank sensor structure, which depicts a pure capacitance characteristic, with a phase of about $-90^{\circ}$. After immobilization of $B$. atrophaeus-spores ( $10^{7}$ colony-forming units (cfu)) the impedance decreases (red lines). This can be attributed as a conductivity enhancement and change of permittivity between the electrode fingers. After the sterilization process a further impedance decrease can be monitored (green lines). This fact can be related to structural modifications of the spores due to the sterilization process, i.e., rupture of the spores and release of ionic substances. These effects lead to further enhancement of the conductivity between the electrode fingers.

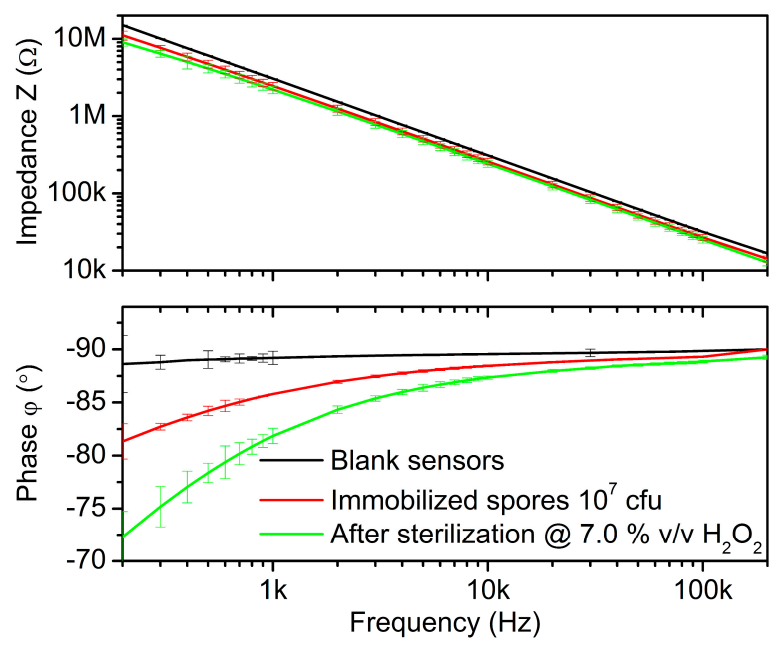

Figure 2. Averaged Bode plot of four independent sensors. Characterizations in vacuum: blank sensor structure (black lines), after immobilization of B. atrophaeus-spores ( $\left.10^{7} \mathrm{cfu} / \mathrm{IDE}\right)$ (red lines) and after sterilization with $\mathrm{H}_{2} \mathrm{O}_{2}$ vapor for $0.3 \mathrm{~s}$ at $\mathrm{c}\left(\mathrm{H}_{2} \mathrm{O}_{2}\right): 7.0 \% v / v, \mathrm{~T}_{\text {gas: }} 240{ }^{\circ} \mathrm{C}, \mathrm{V}_{\text {air: }} 10 \mathrm{~m}^{3} / \mathrm{h}$ (green lines).

\subsection{Morphological Characterizations}

In order to reveal the impact of the sterilization process on the test microorganism, SEM surface characterizations of the spores were performed. As reference non-treated spores were characterized (Figure $3 a$ ) and compared with spores after the sterilization process (Figure $3 b$ ).

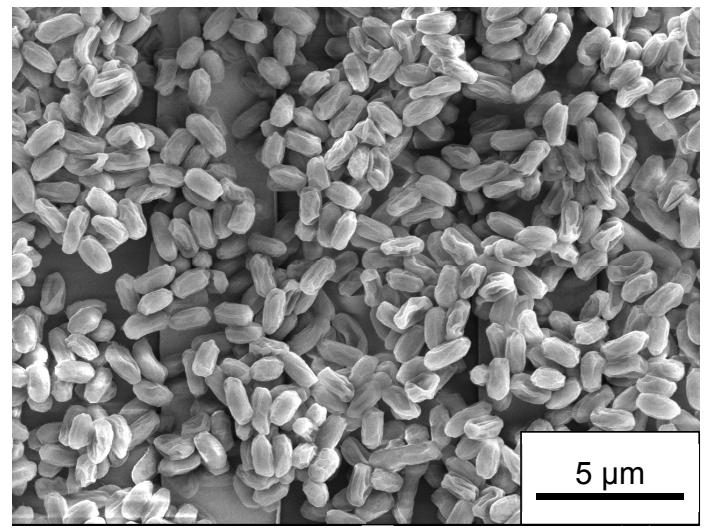

(a)

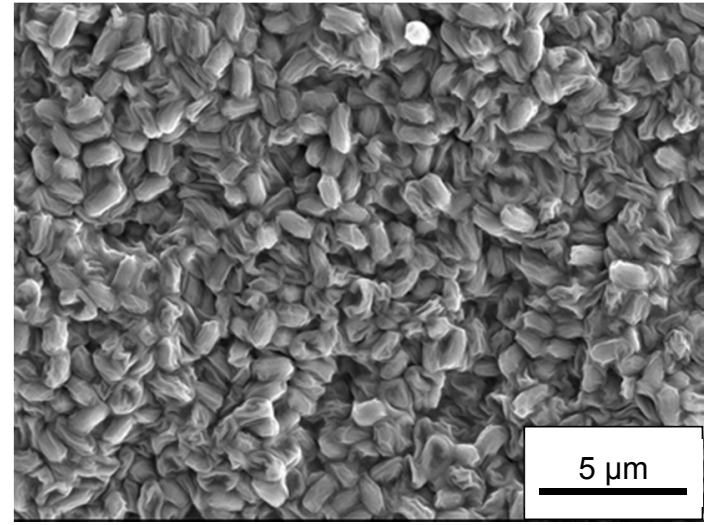

(b)

Figure 3. SEM pictures of (a) non-treated spores and (b) spores after sterilization process in correspondence to the impedimetric characterization at $7.0 \% v / v \mathrm{H}_{2} \mathrm{O}_{2}$. 
The picture of the non-treated spores shows mostly intact spores with an ellipsoidal shape. After the sterilization process the majority of the spores is deformed and collapsed. Moreover, a layer can be observed in which the spores are merged. This layer might be attributed to the disruption of spores and release of spore inner ionic substances. Similar spore disruption has also been observed of plasma-treated spores in [7]. This formed layer after the sterilization process could be related to the impedance decrease observed in the presented impedance measurements.

\section{Conclusions}

In the present work, impedimetric characterizations depicted electrically measurable effects of the sterilization processes on the immobilized spores by means of interdigitated electrodes. In addition, surface characterizations by SEM were applied, which reveal morphological changes caused by the sterilization process. The observed alterations of the spores have been assigned to the detected impedance decrease.

Acknowledgments: This work has been financially supported by the Ministry of Education and Research, Germany (BMBF), Project: “ImpediPack” (Fund. No. 03FH012I3).

Conflicts of Interest: The authors declare no conflict of interest.

\section{References}

1. Toledo, R.T.; Escher, F.E.; Ayres, J.C. Sporicidal properties of hydrogen peroxide against food spoilage organisms. Appl. Microbiol. 1973, 26, 592-597.

2. Ansari, I.A.; Datta, A.K. An overview of sterilization methods for packaging materials used in aseptic packaging systems. Food Bioprod. Process. 2003, 81, 57-65.

3. Oberländer, J.; Jildeh, Z.B.; Kirchner, P.; Wendeler, L.; Bromm, A.; Iken, H.; Wagner, P.; Keusgen, M.; Schöning, M.J. Study of interdigitated electrode arrays using experiments and finite element models for the evaluation of sterilization processes. Sensors 2015, 15, 26115-26127.

4. Oberländer, J.; Bromm, A.; Wendeler, L.; Iken, H.; Durán, M.P.; Greeff, A.; Kirchner, P.; Keusgen, M.; Schöning, M.J. Towards a biosensor to monitor the sterilization efficiency of aseptic filling machines. Phys. Status Solidi A 2015, 212, 1299-1305.

5. VDMA-Fachverbandsschriften. Code of practice: Filling machines of VDMA hygiene class V: Testing the effectiveness of packaging sterilization devices. VDMA-Fachverb. 2008, 6, 1-16.

6. Kirchner, P.; Ng, Y.A.; Spelthahn, H.; Schneider, A.; Henkel, H.; Friedrich, P.; Kolstad, J.; Berger, J.; Keusgen, M.; Schöning, M.J. Gas sensor investigation based on a catalytically activated thin-film thermopile for $\mathrm{H}_{2} \mathrm{O}_{2}$ detection. Phys. Status Solidi A 2010, 207, 787-792.

7. Deng, X.; Shi, J.; Kong, M.G. Physical mechanisms of inactivation of Bacillus subtilis spores using cold atmospheric plasmas. IEEE Trans. Plasma Sci. 2006, 34, 1310-1316.

(c) 2017 by the authors. Licensee MDPI, Basel, Switzerland. This article is an open access article distributed under the terms and conditions of the Creative Commons Attribution (CC BY) license (http://creativecommons.org/licenses/by/4.0/). 